\title{
Estudo comparativo entre anastomoses intestinais com sutura manual e com anel biofragmentável em cães sob a administração de corticosteróides
}

\author{
L. C. Fernandes, D. Matos, M. D. Novelli, S. B. Kim \\ Disciplina de Gastroenterologia Cirúrgica da Unifesp - Escola Paulista de Medicina, São Paulo, SP.
}

\begin{abstract}
RESUMO - ОвJ etivo. Este estudo analisou anastomoses intestinais por sutura manual e por compressão com anel biofragmentável, sob retardo cicatricial pela administração parenteral de corticosteróides.
\end{abstract}

Material e métodos. Vinte cães, entre 15 e 20 kg, foram divididos em grupos controle e teste, este submetido à administração intramuscular de hemissuccinato de hidrocortisona, de 25 a $33 \mathrm{mg} / \mathrm{kg} /$ dia, do 30 o dia pré-operatório ao 70 dia pós-operatório. Em ato cirúrgico, cada animal foi submetido a duas secções cólicas, com anastomoses por sutura manual em plano único extramucoso e por compressão com anel biofragmentável. Os espécimes foram sacrificados sete dias após o procedimento para avaliação das anastomoses.

Resultados. No pós-operatório ocorreram um óbi to no grupo teste e dois óbi tos no grupo controle por fístula não bloqueada nas anastomoses por

\section{NTRODUÇÃO}

A reconstrução digestiva tem constituído objeto de investigação constante na Medicina. Persiste o princípio básico de que a perfeita manipulação dos tecidos e a adequada disposição final dos grupos celulares são essenciais para a perfeita cicatrização.

Vários métodos de sutura digestiva foram empreendidos no decorrer dos séculos. Em 1264, Ruggiero introduziu a prática cirúrgica da sutura digestiva, com fios de seda. Richerand, no século XIX, sustentou a impossibilidade de união de serosa com mucosa, sólido princípio da cirurgia gastrointestinal atual ${ }^{1}$.

Anastomoses passaram a ser realizadas com suturas dispostas em uma ou em duas camadas; contínuas ${ }^{3}$ e em pontos separados ${ }^{4}$; interessando determinadas camadas teciduai $s^{5}$ ou toda a espessura da parede digestiva6; com resultado final invaginante ${ }^{7}$ ou evaginante ${ }^{8}$ das bordas da anastomose. I números materiais foram utilizados para a compressão com anel. Houve, estatisticamente, incidência semel hante de aderências, fístulas, dilatação aferente e obstrução, nos métodos em comparação. À microscopia, houve deficiência de regeneração mucosa nas anastomoses por compressão. Análise histológica por computador evidenciou nas anastomoses por compressão, maior reação inflamatória, maior edema de submucosa e formação de cicatrizes alargadas.

Conclusões. Com o anel biofragmentável, em anastomoses colocólicas sob retardo de cicatrização induzido por corticosteróides, foram obtidos resultados semelhantes aos da sutura manual quanto à incidência de complicações pós-operatórias; 0 anel, entretanto, determi nou pior regeneração mucosa e maior reação inflamatória cicatricial.

UNITERMOS: Anastomose cirúrgica. Cirurgia de cólon e reto. Técnicas operatórias. Corticosteróides. Hidrocortisona.

realização das referidas suturas: colágeno, al godão, seda, linho e diversos el ementos sintéti $\cos ^{9}$.

São consideradas anastomoses seguras, atualmente, as efetuadas em plano único extramucoso, pontos separados ${ }^{10,11}$, com fios monofilamentares de pol i propileno, por exemplo12; e as realizadas por grampeadores, empregando grampos de aço ou titânio ${ }^{13}$.

Têm sido evidenciados, entretanto, efeitos adversos provenientes da permanência de corpos estranhos em suturas, fios cirúrgicos nas suturas manuais e grampos metálicos nas suturas mecânicas. Há referências a maior inflamação local ${ }^{14}$, proliferação celular ${ }^{15}$, carcinogênese ${ }^{16}$, implantação de metástases ${ }^{17}$, mai or formação de aderências peritoneais $^{18}$ e adesão bacteriana ${ }^{19}$.

No intuito de obtenção de uma melhor reparação tecidual têm sido avaliados métodos alternativos de anastomose, como a compressão para a síntese teci dual ${ }^{20}$, sem a permanência de materiais estranhos através dos tecidos dos segmentos digestivos anastomosados. 
Anastomose digestiva por compressão existe desde o século XIX, quando Denans (1826) apresentou dispositivo intraluminar de prata, constituído por dois anéis que promoviam a união de segmentos intestinais em anastomose, por inversão de suas bordas e aposição sero-serosa ${ }^{21}$. Resultados promissores, entretanto, sobrevieram à utilização de dispositivos intraluminares como os preconizados por Murphy (1892)22, dotados de maior canal interno para melhor possibilitar a passagem do conteúdo do tubo digestivo. Esses dispositivos eram constituídos de duas partes acopláveis, entre as quais as paredes em anastomose entravam em processo de compressão e regeneração. O mecanismo, após a cicatrização da anastomose, destacava-se das paredes do órgão e prosseguia em trânsito com as secreções digestivas, sendo eliminado à evacuação do paciente.

O método de compressão não obteve sucesso nas fases iniciais, em virtude dos materiais inadequados utilizados nos dispositivos e do reduzido diâmetro interno dos mecanismos, que impossibilitava adequada passagem do conteúdo digesti vo através do dispositivo e da anastomose ${ }^{23}$. O processo passou a ser revisto na atualidade, revitalizado pel o uso de novos materiais na fabri cação de dispositivos intraluminares de compressão.

Diversos mecanismos foram desenvolvidos: anéis de polipropileno ${ }^{24}$; anéis das pistolas russas AKA ${ }^{25}$,anéis magnéti $\cos ^{26}$ e anéis bi ofragmentáveis (Valtrac $\AA^{27}$. O anel de anastomose biofragmentável constituiu um dos mecanismos desenvolvidos de melhor difusão em estudos experimentais ${ }^{28}$ e clínicos ${ }^{29,30,31}$. É composto de duas metades simétricas que se encaixam sob pressão, mantendo em seu mecanismo as bordas da anastomose sob compressão, com canal interno amplo; biofragmentável, decompõe-se no interior do tubo digestivo, o que facilita sua eliminação. Composto de poliglactina e sulfato de bário, destaca-se da zona de anastomose após os períodos críticos da cicatrização ${ }^{32}$.

Estudos pormenorizados são necessários, no entanto, para a determinação da eficiência do dispositivo mesmo em condições não ideais de cicatrização. O uso experimental sistêmico de corticosteróides em cobaias, por exemplo, vem se caracterizando como eficiente método de simulação de condições desfavoráveis à cicatrização em estudos atuais ${ }^{33,34}$.

Investigação sobre o anel biofragmentável de anastomose sob risco, em condições fisiológicas limítrofes, constituiria análise útil para verificar a efi ciência do dispositivo e a validade da compressão tecidual na realização de anastomoses intesti - nais. Para tanto, desenvolveu-se estudo analisando os resultados precoces obtidos em anastomoses colocólicas por sutura manual e por compressão com anel bi ofragmentável, sob retardo de cicatrização induzido pela administração parenteral de corti costerói des.

\section{MATERIAL E MÉTODO}

A presente investigação foi realizada no Biotério, no Laboratório Central e no Laboratório de Técnica Cirúrgica e Cirurgia Experimental da Universidade Federal de São Paulo - Escola Paulista de Medicina (Unifesp - EPM); e no Laboratório de I nformática Dedicado à Odontologia da Faculdade de Odontologia da Universidade de São Paulo (F OUSP), de setembro de 1995 a dezembro de 1997. Foram utilizados 20 cães sem raça definida, hígidos, peso entre 15 e 20 kg à admissão, sexo masculino.

Foram adotadas normas recomendadas pelo Colégio Brasileiro de Experimentação Animal (Cobea), filiado ao International Council for Laboratory Animal Science (I clas), para o manuseio dos espécimes. Os cães foram divididos al eatoriamente em dois grupos de 10, denominados grupo controle e grupo teste. A randomização foi realizada no início da investigação, por distribuição de postos baseada em tabela de números aleatórios; com esquema de distribuição e procedimentos sob responsabilidade de indivíduo não envolvido com o experimento, consultado após a seleção de cada animal.

O grupo controle (I), não destinado ao recebimento de corticosteróides, foi submetido a intervenção cirúrgica e a posterior sacrifício no 7o dia pós-operatório, para anál ise de anastomoses intestinais efetuadas. Os animais do grupo teste (II) foram submetidos à administração intramuscular diária de hemissuccinato de hidrocortisona, em doses de $500 \mathrm{mg}$ ( 25 a $33 \mathrm{mg} / \mathrm{kg}$ de peso/dia), por um período de 37 dias, com início 30 dias antes do ato cirúrgico estendendo-se até o 7o dia do período pós-operatório, quando se efetuou o sacrifício.

Os animais foram submetidos à análise nutricional nas diversas etapas do experimento. Houve preparo intestinal pré-operatório e esquema profilático com antimicrobiano (cefoxitina, I M, 500 mg no ato anestésico).

A anestesia foi efetuada com acepromazina a $0,2 \%(\mathrm{IM}, 0,2 \mathrm{mg} / \mathrm{kg}$ ) e tiopental sódico (EV , $25 \mathrm{mg} /$ $\mathrm{kg}$ ), com intubação orotraqueal, hidratação (SF 0,9\%, EV, $20 \mathrm{ml} / \mathrm{kg} / \mathrm{h}$ ), ventilação controlada e cateterização vesical.

No ato cirúrgico, secções cólicas completas fo- 


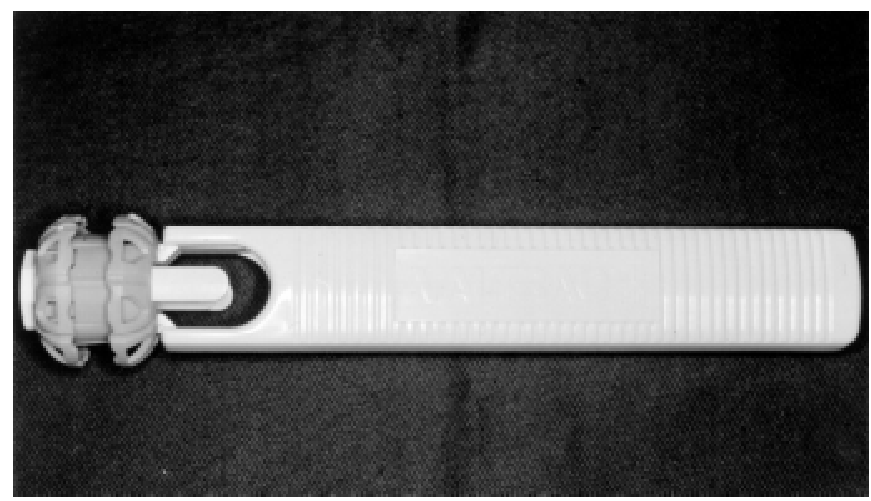

Fig. 1 - Anel anastomóti co bi ofragmentável com aplicador.
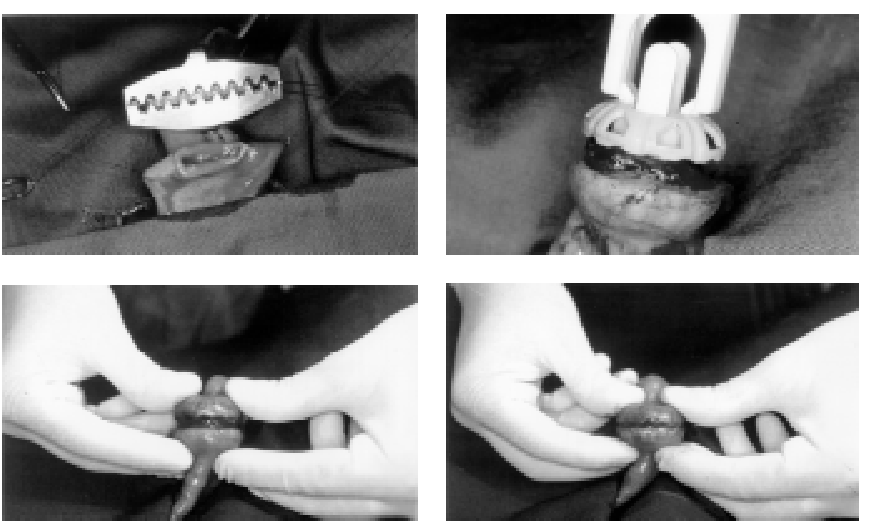

Fig. 2 - Anastomose por compressão com anel biofragmentável. Acima à esquerda, sutura em bolsa nas bordas cólicas; à direita, instalação do dispositivo, fixado pela sutura em bolsa. Abaixo: à esquerda, apreensão do conjunto; à direita, compressão para fechamento do anel.

ram efetuadas a 10 e a $20 \mathrm{~cm}$ da reflexão peritoneal na pelve. Uma das áreas foi submetida a anastomose por sutura manual e a outra foi reparada através de compressão por anel biofragmentável. A disposição dos dois métodos de síntese foi alternada entre os animais, aleatoriamente.

Nas anastomoses por compressão utilizou-se anel biofragmentável, composto de ácido poliglicól i co (87,5\%) e sulfato de bári o (12,5\%), de diâmetro externo de 25, 28 ou $31 \mathrm{~mm}$, com gap de 2,0 mm entre as peças componentes após o fechamento do anel (Fig.1). Os segmentos intestinais foram posicionados no anel com o fechamento de suturas em bolsa previamente confeccionada; a compressão extrínseca dos componentes do anel promovendo seu fechamento e a compressão das bordas dos segmentos cólicos, em anastomose término-terminal (Fig.2).

A anastomose por sutura manual, colocólica término-terminal em plano único, foi realizada através de pontos separados, extramucosos, a distâncias de $3 \mathrm{~mm}$, com nós exteriores à luz do órgão, utilizando-se fio de polipropileno monofilamentar 4-0.
A síntese da parede abdominal foi realizada por sutura em massa contínua com fio de polipropileno 0 ; síntese cutânea com pontos simples invertidos, fio de nylon 00 .

Oferecimento de dieta foi realizado a partir do primeiro dia pós-operatório, com avaliação clínica diária e verificação de eliminação do anel biofragmentável nas fezes.

No sacrifício dos espécimes no sétimo dia pósoperatório, foi feita a verificação das condições intracavitárias e efetuada a excisão dos segmentos anastomosados em bloco; o sacrifíci o foi obtido com administração $\mathrm{EV}$ de solução de $\mathrm{KCl}$ a $19,1 \%, 20 \mathrm{ml}$.

As peças cirúrgicas excisadas foram lavadas e anal isadas macroscopicamente, com estudo da situação do anel biofragmentável.

A avaliação histológica convencional foi realizada em cortes corados por hematoxilina-eosina. As lâminas histológicas geraram imagens que foram digitalizadas e analisadas por programa DIRACOM-3 de análise gráfica. Por subtração de imagens realizou-se análise quantitativa da reação inflamatória tecidual existente, por cálculo da presença relativa de infiltrado intersticial, de matriz protéica e de celularidade na cicatriz. Também foram realizadas medições lineares nas imagens digitalizadas, determinando a espessura do tecido de reparação e o edema de submucosa na região.

Foram utilizados na avaliação dos resultados: teste de Wilcoxon; teste de Friedman complementado pel o teste de comparações múl ti plas; teste de Fisher; teste de McNemar; teste de Mann-Whitney e teste " $t$ " de Student. Fixou-se em 5\% ( $\alpha \leq$ $0,05)$ o nível para valores significantes.

\section{RESULTADOS}

Quanto à análise nutricional não houve, em regra geral, variação dos parâmetros nos espécimes do grupo controle, nas diversas fases do experimento. No grupo teste houve decréscimo significante das variáveis analisadas. Os espécimes submetidos a administração de corticosteróides sofreram intervenção cirúrgica com quadro nutricional mais distante da normalidade ${ }^{36}$ que os cães do grupo controle (Tabela 1).

Cinco espécimes do grupo teste (50\%) apresentaram efeitos colaterais decorrentes da administração de corticosteróides, tais como: diarréia, enterorragia e vômitos.

O preparo intestinal evidenciado no ato cirúrgico foi semelhante nos espécimes de ambos os grupos.

No pós-operatório, infecção e deficiência de ci- 


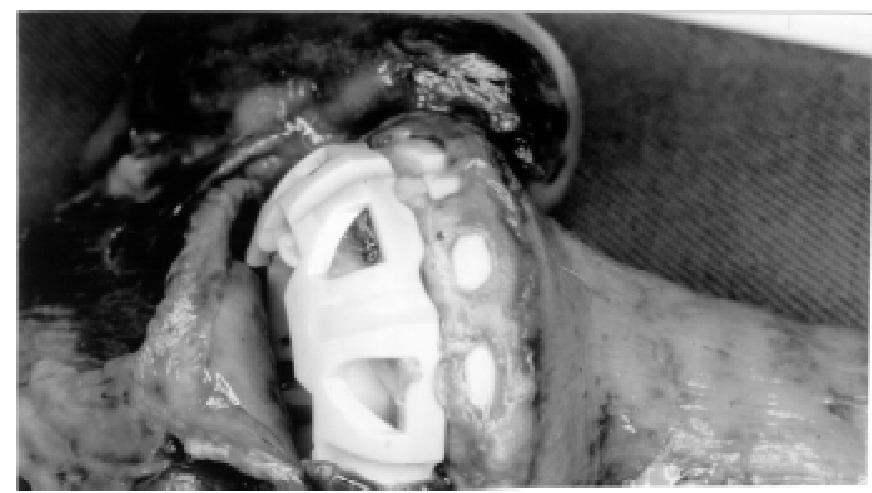

Fig. 3 - Deiscência de anastomose colocólica por compressão com anel, cão 9, grupo teste, 50 P.O.; necrose estendendo-se além dos tecidos mantidos sob compressão pelo dispositivo biofragmentável.

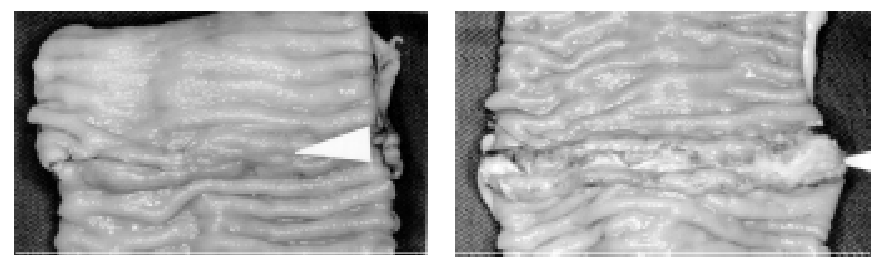

Fig. 4 - F ace mucosa das anastomoses col ocól icas do cão 11, grupo teste, 7ㅇ P.O. À esquerda: anastomose por sutura manual, com epitel ização sati sfatória; à direita: anastomose por compressão com anel bi ofragmentável com ulceração de mucosa e persistência dos fios da cutura em bolsa.

catrização na incisão cirúrgica foram incidentes em maior proporção no grupo teste, com diferença estatisticamente significante.

Durante o experimento, quatro espécimes apresentaram óbito (Tabela 2).

$\mathrm{Na}$ avaliação macroscópica das anastomoses, no sacrifício dos espécimes, observou-se maior incidência de alterações nas anastomoses efetuadas por compressão com anel biofragmentável (Tabela 3) (Fig.3).

Não houve diferença estatística detectável quanto à extensão e à constituição das aderências desencadeadas pelos métodos de anastomose em estudo.

O dispositivo bi ofragmentável, no sacrifício, encontrou-se fixo à linha de anastomose na maior parte dos casos. A maioria dos anéis destacados foi encontrada em estado íntegro, não fragmentado.

Anastomoses com utilização do anel foram desfavoráveis quanto a presença de esporão de mucosa e ulceração (Fig. 4). Na análise histológica convencional foram identificados granulomas de corpo estranho com maior freqüência nas anastomoses por anel biofragmentável.

A digitalização de imagens histológicas (Fig. 5) demonstrou que as anastomoses intestinais por compressão com anel suscitaram respostas infla-
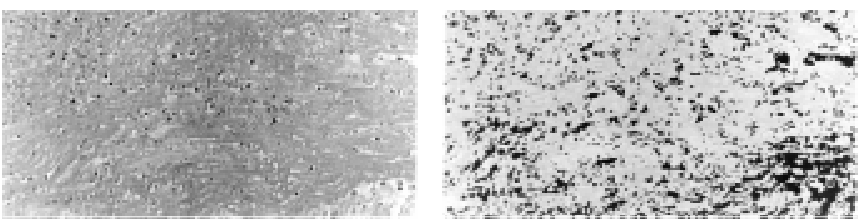

Fig. 5 - Avaliação histológica por digitalização de imagens (Programa DIRACOM-\#). À esquerda: zona cicatricial de anastomose colocólica por compressão com anel, cão 17, grupo teste 70 P.O. À direita: subtração de imagens referentes a interstícios. Aumento 200x.

matórias mais importantes, com maior edema intersticial na cicatriz: 7,12\% e 7,35\% nas anastomoses por sutura manual dos grupos controle e teste; $11,02 \%$ e $12,13 \%$ nas anastomoses por compressão com anel nos grupos controle e teste, respectivamente.

Medidas lineares evidenciaram que a compressão com anel provocou maior edema na submucosa adjacente à zona de anastomose e formação de cicatrizes mais alargadas (Tabela 4).

\section{DISCUSSÃO}

A presente pesquisa experimental possibilitou avaliações da cicatrização através de parâmetros de impossível obtenção em estudos clínicos, analisando os tecidos em anastomose em toda a sua extensão ${ }^{37}$. A randomização permitiu minimizar diferenças entre os subgrupos. A realização dos dois métodos de anastomose intestinal em cada um dos espécimes permitiu que as técni cas sob anál ise fossem expostas aos mesmos fatores intervenientes externos em cada animal ${ }^{38}$.

Análises físicas das anastomoses, pressão e força de tração para rompimento teci dual ${ }^{39,40}$, seriam úteis. No entanto, peças cirúrgicas utilizadas em testes físicos seriam inadequadas para a realização posterior de análises histológi cas precisas.

A administração de corticosterói des afeta a cicatrização intestinal nos sete dias iniciais do período pós-operatório ${ }^{34,41}$; estudos em fases posteriores não analisariam processos cicatriciais com alteração de evolução. O uso de corticosteróides evidenciou-se adequado para cirurgia experimental, sem provocar el evada letalidade e promovendo alterações sistêmicas e de cicatrização tecidual.

É controverso o papel dos corticosteróides no metabolismo animal, havendo consenso na manifestação de estados anaból i cos e catabóli icos simultaneamente $e^{42}$. Valores de hemoglobina, hematócrito, contagem eritrocitária, concentração sérica de proteínas totais e de albumina decresceram no grupo teste. Terapêutica com corticosteróides cursa com el evação do volume eritrocitário e da massa hemoglobínica sangüínea; no entanto, estudos 


\begin{tabular}{|c|c|c|c|}
\hline Parâmetros & Cães do grupo controle & Cães do grupo teste & Valores normais 50 \\
\hline Peso (Kg) & 16,44 & 14,79 & - \\
\hline Hemoglobina (g/dl) & 11,54 & 9,62 & $12,0-18,0$ \\
\hline Hematócrito (vol\%) & 33,6 & 28,6 & $37,0-55,0$ \\
\hline № de eritrócitos & 4.900 .000 & 4.100 .000 & $5.500 .000-8.500 .000$ \\
\hline \multicolumn{4}{|l|}{$(\mathrm{n} \% / \mu \mathrm{l})$} \\
\hline Proteínas totais (g/dl) & 6,7 & 5,6 & $5,2-7,0$ \\
\hline
\end{tabular}

\begin{tabular}{|c|c|c|c|}
\hline Cão & Grupo & Período do óbito & Causa do óbito \\
\hline$L-13$ & teste & pré-operatório & $\begin{array}{l}\cdot \text { colite(?) } \\
\text { - peritonite }\end{array}$ \\
\hline L-09 & teste & 5 P.O. & $\begin{array}{l}\text { - deiscência da anastomose } \\
\text { pelo anel biofragmentável } \\
\text { - peritonite }\end{array}$ \\
\hline L-02 & controle & 4으. P.O. & $\begin{array}{l}\text { - deiscência da anastomose } \\
\text { pelo anel biofragmentável } \\
\text { - peritonite }\end{array}$ \\
\hline L-14 & controle & 5 P.O. & $\begin{array}{l}\text { - deiscência da anastomose } \\
\text { pelo anel biofragmentável }\end{array}$ \\
\hline
\end{tabular}

com emprego de altas doses em animais têm reveIado inibição da eritropoese ${ }^{42}$. E m relação ao metabolismo protéico, registra-se manutenção dos valores de proteína sérica total com o emprego experimental de corticosteróides em baixa dosagem ${ }^{43}$; utilização em doses maiores revelam queda significante da concentração protéi ca plasmática e intestinal ${ }^{44}$.

Os espécimes do grupo teste foram submetidos ao ato cirúrgico em condições sistêmicas deteri oradas em relação aos animais do grupo controle. Efeitos colaterais característicos contribuíram para mai or desgaste nos espécimes do grupo teste. Houve predomínio de intercorrências na cicatrização cutânea dos espécimes do grupo teste, com inadequada adesão dos tecidos e el evado índice de complicações sépticas locais.

O preparo intestinal evidenciado no ato cirúrgico foi semelhante nos grupos, não constituindo elemento de interferência na evolução da cicatrização das anastomoses intestinais.

Um dos espécimes apresentou óbito pré-operatório por colite. Os demais óbitos transcorreram entre o quarto e o quinto dia pós-operatório, fase de modificações no colágeno tecidual das anastomoses intestinais $\mathrm{s}^{45,46}$ e risco de ocorrência de fístulas anastomóticas ${ }^{47}$. Houve peritonite devido a deiscência em anastomoses por compressão com anel biofragmentável. Em um dos casos evidenciou-se obstrução do anel por pêlos deglutidos, com ruptura tecidual proximal por distensão intestinal. Nos outros casos houve necrose das bordas teciduais, que se estendia além da zona circunscrita pelo mecanismo do anel.

A maioria dos estudos experimentais não tem relatado ocorrência de fístulas anastomóticas pelo emprego do anel bi ofragmentável ${ }^{27,28}$. No entanto, há registro experimental de fístulas em anastomoses colorretais com anel após irradiação ${ }^{48}$ e fístulas em anastomoses esôfago-esofágicas por escarificação traqueal ${ }^{49}$.

Trabalhos clínicos têm atestado segurança de utilização do anel em variadas situações clínicas e diversos sítios anatômi $\cos ^{29,30,31}$. Certos estudos, entretanto, apresentam índice de fístulas anastomóti cas representativo em intervenções com uso do anel anastomótico bi ofragmentável ${ }^{50}$.

O encontro de dilatação aferente nas anastomoses por compressão no experimento evidencia que o anel fragmentável pode oferecer obstáculo à passagem do conteúdo intestinal ${ }^{51}$. Em protocol os clínicos houve registro de sinais de suboclusão e obstrução intestinal ${ }^{52,53}$.

A freqüência de processos aderenciais e sua intensidade foi semel hante nas anastomoses pelos métodos em comparação. Há controvérsia sobre tais parâmetros ${ }^{18,54}$.

O anel biofragmentável foi encontrado geralmente fixo às anastomoses intestinais em que foi utilizado. Quando destacado, apresentou integri- 


\begin{tabular}{|c|c|c|c|c|c|c|c|c|}
\hline \multirow{3}{*}{ Eventos } & \multicolumn{4}{|c|}{ Sutura } & \multicolumn{4}{|c|}{ Anel Biofragmentável } \\
\hline & \multicolumn{2}{|c|}{$\begin{array}{l}\text { G. controle } \\
\quad(n=10)\end{array}$} & \multicolumn{2}{|c|}{$\begin{array}{l}\text { G. teste } \\
\left(n^{\prime}-9\right)\end{array}$} & \multicolumn{2}{|c|}{$\begin{array}{l}\text { G. controle } \\
\quad(n=10)\end{array}$} & \multicolumn{2}{|c|}{$\begin{array}{l}\text { G. teste } \\
\left(n^{\prime}=9\right)\end{array}$} \\
\hline & f. & $\%$ & f. & $\%$ & f. & $\%$ & f. & $\%$ \\
\hline - Aderências & 8 & 80 & 7 & 77,8 & 10 & 100 & 6 & 66,7 \\
\hline \multicolumn{9}{|c|}{ - Fístula não bloqueada } \\
\hline + peritonite & 0 & 0 & 0 & 0 & 2 & 20 & 1 & 11,1 \\
\hline - Dilatação aferente & 0 & 0 & 0 & 0 & 3 & 30 & 2 & 22,2 \\
\hline \multicolumn{9}{|l|}{ - Obstrução da } \\
\hline anastomose & 0 & 0 & 0 & 0 & 1 & 10 & 0 & 0 \\
\hline
\end{tabular}

Tabela 4-Espessura média em um do tecido cicatricial e da submucosa adjacente às anastomoses intestinais por sutura manual e por compressão com anel biofragmentável, grupos controle e teste, sétimo P.O.

\begin{tabular}{|c|c|c|c|c|}
\hline \multirow[t]{2}{*}{ Parâmetros } & \multicolumn{2}{|c|}{ Grupo controle } & \multicolumn{2}{|c|}{ Grupo teste } \\
\hline & Sutura manual & Anel biofragmentável & Sutura manual & Anel biofragmentável \\
\hline $\begin{array}{l}\text { Espessura } \\
\text { cicatricial }^{*}\end{array}$ & 957,26 & $2.000,09$ & 548,82 & $1.267,72$ \\
\hline $\begin{array}{l}\text { Espessura de } \\
\text { submucosa* }\end{array}$ & 903,99 & $1.903,46$ & 722,16 & $1.351,33$ \\
\hline
\end{tabular}

dade de suas partes constituintes. Quando a fragmentação do anel ocorre na luz intestinal, seu deslocamento dos tecidos da anastomose provavelmente se deve à necrose tecidual marginal. Teoricamente, há necrose tecidual controlada e autolimitada na cicatrização por emprego do anel biofragmentável ${ }^{27}$. No entanto, necrose tecidual é evento variável e imprevisível, podendo originar maior reação inflamatória, pi ores resultados cicatriciais, necrose não controlada e estendida, deiscências e morbi mortal idade maior, como houve nesta investigação. Não há necrose asséptica no interior do tubo digestivo; e há dificuldades para a limitação desse processo nos tecidos situados no interior de dispositivos de compressão em anastomoses intestinais ${ }^{55}$. Talvez, geralmente o desprendimento do anel dos tecidos se processe por necrose tecidual e não por fragmentação das partes componentes.

Microscopicamente, alterações de mucosa foram eventos mais freqüentes nas anastomoses por anel: esporão de mucosa e ulceração.

Houve maior presença de granulomas de corpo estranho nas anastomoses por compressão. Pelas características do método, não deveria haver a presença de corpos estranhos na região cicatricial. É possível que o fio da sutura em bolsa das bordas constitua a origem das reações de corpo estranho identificadas. A anastomose por compressão com anel biofragmentável não é desprovida da presença de corpos estranhos na região de cicatrização nas fases precoces de reparação. Hardy et al. (1985), na descrição original do anel anastomótico biofragmentável referem a permanência do fio da sutura em bolsa no sítio de união tecidual por 22 dias após a intervenção cirúrgica ${ }^{27}$.

A aval iação histológica por digital ização de imagens permitiu, ao contrário de subjetivas interpretações tradicionais, a realização de medidas precisas do edema intersticial e de distâncias lineares na cicatriz. Houve maior reação inflamatória nas anastomoses efetuadas por compressão com anel. O anel deveria promover cicatrização digestiva com menor reação inflamatória pela inexistência de corpos estranhos na linha de sutura. É factível que a ocorrência de necrose nos tecidos marginais, ou que a presença de corpos estranhos na linha de sutura representados pelo fio da sutura em bolsa nas bordas em anastomose, tenham provocado maior reação inflamatória local.

A espessura do tecido cicatricial e o edema de submucosa foram maiores nas anastomoses promovidas por compressão com anel biofragmentável.

Seria a compressão tecidual um método de anastomose intestinal capaz de proporcionar cicatrização superior à obtida por métodos de sutura? 
O anel anastomótico biofragmentável foi desenvolvido com o intuito de promover perfeita anastomose por compressão ${ }^{28}$. É método padronizado e de rápida execução ${ }^{18}$. Confere resistência mecânica à anastomose nas fases precoces da cicatrização; a ausência de corpos estranhos na cicatriz possibilitaria melhores resultados de regeneração ${ }^{28}$.

Há, no entanto, desvantagens. É de difícil utilização em certas regiões anatômicas, principalmente extraperitoneais ${ }^{56}$. É necessária disponibilidade de anéis de di versos diâmetros e "gaps" para adequada utilização ${ }^{32}$. As metades do dispositivo não são destacáveis entre si, o que dificulta sua instalação $0^{57}$. O anel provocou maior reação inflamatória e desfavorável regeneração da mucosa nesta investigação.

Talvez o anel bi ofragmentável, em sua concepção atual, não constitua o dispositivo ideal. M odificações e aperfeiçoamentos são necessários para que mecanismos de compressão tecidual possam suplantar os métodos de sutura nas anastomoses intestinais.

\section{CONCLUSÕES}

Em anastomoses colocólicas sob retardo de cicatrização induzido por corticosteróides a sutura manual e a compressão com anel biofragmentável foram similares quanto à ocorrência de aderências, fístulas, dilatação aferente e obstrução; a compressão por anel propiciou regeneração mucosa deficiente e maior reação inflamatória cicatricial.

\section{SUMMARY}

\section{Comparative study of intestinal anastomo- ses with manual suture and biofragmentable ring in dogs under corticosteroid adminis- tration.}

BACKGROUND. This study analyzed intestinal anastomoses by manual suture and by compression with biofragmentable ring under delay of cicatrization administering parenteral corticoids.

Material AND METHODS. Twenty dogs were divided into two groups: control and test, the latter submitted to intramuscular administration of hydrocortisone hemisuccinate, 25 to $33 \mathrm{mg} / \mathrm{kg} /$ day, on the $30^{\text {th }}$ preoperative and $7^{\text {th }}$ postoperative days. During surgery, each animal underwent two col on sections with anastomosis by manual suture in a single extramucous plane and compression with biofragmentable ring. The animals were sacrificed 7 days after the procedure to evaluate the anastomoses.

Results. In the postoperative period, one death occurred in the test group and two in the control group, caused by nonblocked fistula in the anastomoses by ring compression. Statistically, there was a similar incidence of adherences, fistulas, afferent dilatation and obstruction using comparison methods. On microscopy, deficiency in mucous regeneration of the anastomoses by compression was observed. Computerized histological analysis evidenced in the anastomoses by compression, a greater inflammatory reaction, greater edema of the submucous membrane and enlarged scars.

Conclusions. It was concluded that, with the bi ofragmentable ring, in colonic anastomosis under delay of cicatrization induced by corticoids, similar results to manual suture regarding to postoperative complications incidence were obtained; ring, however, determined worse mucous regeneration and greater cicatricial inflammatory reaction. [Rev Ass Med Bras 2000; 46(2); 113-20]

KEY WORDS: Anastomosis surgical. Colon and rectal surgery. Surgery operative. Adrenal cortex hormones. Hydrocortisone

\section{REFERÊ NCIAS BIBLIOGRÁFICAS}

1. Catalano, F.E. - Enterorrafias: estudo crítico experimental. Buenos Aires, 1941. Tese de doutorado - F acultad de Ciencias Médicas - Universidad Nacional de Buenos Aires

2. Halsted, W.S. - Circular suture of the intestine. Am. J . Med. Sci., 1887, 94:436-61.

3. Connel, M.E. - An experimental contribution looking to an improved technique in enterorraphy, whereby the number of knots is reduced to two, or even one. Med. Rec., 1892, 42:335-7.

4. Gorodiche, J.\& J ourdan,P. - Anastomoses digestives en un plan de suture. Sem. Hop. Paris, 1951, 4:3740-7.

5. Sako,Y. \& Wangensteen, O.H. - Experimental studies on intestinal anastomoses. Surg. Forum, 1951, 2:117-123.

6. Gambee, L.P. - A single-layer open intestinal anastomosis applicable to the small as well as the large intestine. West. J . Surg., 1951, 59:1-5.

7. Lembert, A. - Memoire sur l'enterorraphie avec la description d'un procédé nouveau pour pratiquer cette opération chirurgicale. Rep. Gen. Anat. Physio. Path., 1826, 2:100-7.

8. Getzen, L.C.; Roe, R.D.; Holloway, C.K. - Comparative study of intestinal anastomotic healing in inverted and everted closures. Surg. Gynec. Obstet., 1966, 123:1219-27.

9. Fagundes, D.J . \& Kharmandayan, P. - O fio cirúrgico. Acta Cir. Bras., 1991, 6:177-81.

10. Faria, P.A.J . - Sutura gastro-intestinal em plano único extramucoso e em 2 planos, um total e um seromuscular invaginante: estudo experimental no cão. São Paulo,1972. Tese Doutorado - Escola Paulista de Medicina

11. Warde, P.J . - Anastomose colorretal experimental em um e dois planos de sutura: estudo comparativo. São Paulo, 1972. TeseDoutorado-FaculdadedeMedicina daUniversidadedeSãoPaulo

12. Hastings, J .C.; Winkle J R., W.; Barker, E.; Hines, D.; Nichols,W.- Effect of suture materials on healing wounds of the stomach and colon. Surg. Gynecol. Obstet., 1975, 140:701-7.

13. Steichen, F.M. \& Ravitch, M.M. - Instrumentos de grampeamento contemporâneo e técnicas básicas de sutura mecânica. Clin. Cir. Am. Norte, 1984, 3:447-462.

14. Postlehwait, R.W. - Long term comparative study of nonabsorbable sutures. Ann. Surg., 1970, 171:892-8. 
15. McCue, J.L. \& Phillips, R.K.S. - Celular proliferation at sutured and sutureless colonic anastomoses. Dis.Col on Rectum, 1993, 36:468-74.

16. Phillips, R.K.S. \& Cook, H.T. - Effect of steel wire sutures on the incidence of chemically induced rodend colonic tumours. Br. J . Surg., 1986, 73:671-4.

17. McGregor, J .R.; Galloway, D.J .; McCulloch, P.; George, W.D. - Anastomotic suturematerials and implantation metastases: an experimental study. Br. J . Surg., 1989, 76:331-4.

18. Matos, D.; Saad, S.S.; Franceschi J r., O.; Barreto, E.P.; Novelli, M.D. - Estudo experimental comparativo entre anastomose colocólica com sutura manual e com anel biofragmentável. Rev. Ass. Med. Bras., 1993, 39:201-6.

19. Katz, S.; Izhar, M.; Mirelman, D. - Bacterial adherence to surgical sutures: a possiblefactor in suture induced infection. Ann. Surg., 1981, 194:35-41.

20. McCue, J .L. \& Phillips, R.K.S. - Sutureless intestinal anastomoses. Br. J . Surg., 1991, 78:1291-6.

21. Denans, F.N. - Nouveau procédé pour la guérison des plaies des intestins. Recueil de la Société royale de Médecine de Marseille [Séance de 24 fev. 1826, rédigé par M.P.Roux]. Marseille, I mprimerie d'Archard,1827,Tomel :127-131.

22. Murphy, J .B. - Cholecysto-intestinal, gastro-intestinal, entero-intestinal anastomosis, and approximation without sutures. Med. Rec., 1892, 42:665-76.

23. Matos, D. - Anastomose intestinal por compressão com o dispositivo russo AKA: análise dos resultados clínicos, radiológicos e endoscópicos. São Paulo, 1996. Tese - Livre Docência - Universidade Federal de São Paulo - Escola Paulista de Medicina.

24. Rosati, R.; Rebuffat, C.; Pezzuoli, G. - A new mechanical device for circular compression anastomosis: preliminary results of animal and clinical experimentation. Ann. Surg., 1988, 207:245-52.

25. Matos, D. \& Phillips, R.K.S. - Experiência inicial comodispositivo de anastomose intestinal por compressão AKA-2 e AKA-4 em cirurgia colorretal. Rev. Ass. Med. Bras., 1993, 39:8-11.

26. J ansen, A.; Keeman, J .N.; Davies, G.A.G.; Klopper, P.J . Early experience with magnetic rings in resection of the distal colon. Neth. J . Surg., 1980, 32:20-7.

27. HardyJ r., T.G.; Pace, W.G.; Maney, J .W.; Katz, A.R.; Kaganov, A.L. - A bi ofragmentablering for sutureless bowel anastomosis: an experimental study. Dis. Col on Rectum, 1985, 28:484-90.

28. Maney, J .W.; Katz, A.R.; LI, L.K.; Pace, W.G.; Hardy, T.G. Biofragmentable bowel anastomosis ring: comparative efficacy studies in dogs. Surgery, 1988, 103:56-62.

29. Bubrick, M.P.; Corman, M.L.; Cahill, C.J . et al. - Prospective, randomized trial of the biofragmentable anastomosis ring. Am. J . Surg., 1991, 161:136-43.

30. Corman, M.L.; Prager, E.D.; HardyJ r., T.G.; Bubrick, M.P. Comparison of the Valtrac biofragmentable anastomosis ring with conventional suture and stapled anastomosis in colon surgery: results of a prospective randomized clinical trial. Dis. Col on Rectum, 1989, 32:183-7.

31. Gullichsen, R.; Havia, T.; Ovaska, J .; Ramtala, A. - Colonic anastomosis using the bi fragmentable anastomotic ring and manual suture: a prospective, randomized study. Br.J . Surg., 1992, 79:578-80.

32. Hardy J r., T.G.; Aaguilar, P.S.; Stewart, W.R.C. et al - I nitial clinical experience with a biofragmentable ring for sutureless bowel anastomosis. Dis. Col on Rectum, 1987, 30:55-61.

33. Aszodi, A. \& Ponsky, J .L. - Effects of corticosteroid on the healing bowel anastomosis. Am. Surg., 1984, 50:546-8.

34. Furst, M.B.; Stromberg, B.V.; Blatchford, G.J .; Christensen, M.A.; Thorson, A.G. - Colonic anastomoses: bursting strength after corticosteroid treatment. Dis. Col on Rectum, 1994, 37:12-5.

35. Goldenberg, S. - Normas. Acta Cir. Bras., 1993, 8(supl.1):8-24.
36. Kirk, R.W. - Atualização terapêutica veterinária. 2.ed. São Paulo, Ed. Manole, 1988. 1688p.

37. Koruda, M.J . \& Rolandelli, R.H. - Current research review: experimental studies on the healing of col onic anastomoses. J . Surg. Res., 1990, 48:504-15.

38. Donoso, A.C. Estudo comparativo em cães entre anastomoses col ocól i cas revesti das ou não revestidas internamente por um dreno de Penrose. São Paulo, 1995. Tese - Mestrado - Universidade F ederal de São Paulo - Escola Paulista de Medicina.

39. Ballantyne, G.H. - Intestinal suturing: review of the experimental foundations for traditional doctrines. Dis.Colon Rectum, 1983, 26:836-43.

40. Hendriks, T. \& Mastboom, W.J .B. - Healing of experimental intestinal anastomoses: parameters for repair. Dis. Colon Rectum, 1990, 33:891-901.

41. MartinsJ r., A.M.; Guimarães, A.S.; Ferreira, A.L. - Efeito dos corticosteróides na cicatrização de anastomoses intestinais. Acta Cir. Bras., 1992, 7:28-30.

42. Baxter, J.D. \& Forsham, P.H. - Tissue effects of glucocorticoids. Am. J . Med., 1972, 53:573-589.

43. Howes, E.L.; Plotz, C.M.; Blunt, J .W.; Tagan, C. - Retardation of wound healing by cortisone. Surgery, 1950, 28:177-81.

44. Shoji, S. - Effect of exogenous cortisone on amino acid metabolism in rats. Int. J. Biochem., 1990, 22:83-7.

45. Cronin, K.; J ackson, D.S.; Dunphy, J .E. - Changing bursting strength and collagen content of the healing colon. Surg. Gynecol. Obstet., 1968, 126:747-53.

46. J iborn, H.; Ahonen, J .; Zederfeldt, B. - Healing of experimental colonic anastomoses: I . bursting strength of the col on after left col on resection and anastomosis. Am. J . Surg., 1978, 136:587-94.

47. Hawley, P.R. - Causes and prevention of colonic anastomotic breakdown. Dis. Col on Rectum, 1973, 16:272-7.

48. Smith, A.D.; Bubrick, M.P.; Mestitz, S.T. et al. - Evaluation of the bi ofragmentable anastomotic ring following preoperative irradiation to the rectosigmoid in dogs. Dis. Colon Rectum, 1988, 31:5-9.

49. Polonio, B. - Anastomoses esôfago-esofági cas cervicais término-terminais por invaginação e com anel biofragmentável: estudo comparativo em cães. São Paulo, 1994. Tese - Doutorado - Escola Paulista de Medicina

50. Luukkonen, P.; J ärvinrn, H.J .; Haapiainen, R. - Early experience with biofragmentable anastomosis ring in colon surgery. Acta Chir. Scand., 1990, 156:795-9.

51. Gullichsen, R.; Ovaska, J .; Brasken, P.; Havia, T.; Ekfors, T. Large bowel anastomosis in dogs: a comparative study with thefragmentablering, manual sutureand mechanical stapler. Res. Surg., 1991, 3:162-5.

52. Cahill, C.J . - Multicentre european prospective randomized trial of theValtracbioabsorbableanastomosis ring. In: ENGEMAN, R. \& THIEDE, A., ed. - Intestinal anastomoses with bi oabsorbable anastomosis rings. Berlin, Springer-Verlag, 1993. p.31-5.

53. Deitz, W. - Occlusion ileus due to blood clots following right hemicoletomy: a rare complication. In: Eengeman, R. \& Thiede, A., ed. -I ntestinal anastomoses with absorbableanastomosis rings. Berlin, Springer-Verlag, 1993. p.53-7.

54. Polonio, B. - Estudo comparativo entre as anastomoses colorretais em pl ano úni co extramucoso ecom anel bi ofragmentável no reto extraperitoneal de cães. São Paulo, 1992. Tese Mestrado - Escola Paulista de Medicina

55. Senn, N. - Enterorraphy; its history, technique and present status. J AMA, 1893, 21:215-35.

56. Wood, J.S. \& F rost, D.B. - Results using the bi ofragmentable anastomotic ring for colon anastomosis. Am. Surg., 1993, 59:642-4.

57. Cahill, C.J .; Betzler, M.; Gruwez, J .A. et al. - Sutureless large bowel anastomosis:european experience with the biofragmentable anastomosis ring. Br. J . Surg., 1989, 76:344-7. 